TRANSACTIONS OF THE

AMERICAN MATHEMATICAL SOCIETY

Volume 362, Number 9, September 2010, Pages 4481-4495

S 0002-9947(10)05054-3

Article electronically published on April 6, 2010

\title{
HYPERSURFACES CUTTING OUT A PROJECTIVE VARIETY
}

\author{
ATSUSHI NOMA
}

\begin{abstract}
Let $X$ be a nondegenerate projective variety of degree $d$ and codimension $e$ in a projective space $\mathbb{P}^{N}$ defined over an algebraically closed field. We study the following two problems: Is the length of the intersection of $X$ and a line $L$ in $\mathbb{P}^{N}$ at most $d-e+1$ if $L \nsubseteq X$ ? Is the scheme-theoretic intersection of all hypersurfaces of degree at most $d-e+1$ containing $X$ equal to $X$ ? To study the second problem, we look at the locus of points from which $X$ is projected nonbirationally.
\end{abstract}

\section{$\S 0$. INTRODUCTION}

Let $X \subseteq \mathbb{P}^{N}(N=n+e)$ be a projective variety of dimension $n$, degree $d$, and codimension $e$ over an algebraically closed field $\mathbb{k}$. We always assume that $X$ is nondegenerate in $\mathbb{P}^{N}$; i.e., $X$ is not contained in any hyperplane in $\mathbb{P}^{N}$. Let $m$ be a positive integer. We say that $X$ is $m$-regular if its ideal sheaf $\mathcal{I}_{X / \mathbb{P}^{N}}$ satisfies $H^{i}\left(\mathbb{P}^{N}, \mathcal{I}_{X / \mathbb{P}^{N}} \otimes \mathcal{O}_{\mathbb{P}^{N}}(m-i)\right)=0$ for all $i>0$ (see [16], Lecture 14). By $\mathrm{E}_{m}(X)$, we denote the scheme-theoretic intersection of all hypersurfaces in $\mathbb{P}^{N}$, containing $X$, of degree at most $m$. We consider the following conditions on $X$ :

$\left(A_{m}\right) l(X \cap L):=\operatorname{length}\left(\mathcal{O}_{X \cap L}\right) \leq m$ for each line $L$ in $\mathbb{P}^{N}$ with $L \nsubseteq X$;

$\left(B_{m}\right) X=\mathrm{E}_{m}(X)$

$\left(C_{m}\right) X$ is $m$-regular.

The purpose here is to study $\left(A_{m}\right)$ and $\left(B_{m}\right)$ for $m=d-e+1$. To study $\left(B_{m}\right)$, we also look at the structure of the locus, denoted by $\mathrm{B}(X)$ and $\mathrm{C}(X)$ (see $(0.1)$ ), of points from which $X$ is projected nonbirationally.

First we briefly look at the three conditions. It is well-known that $\left(C_{m}\right)$ implies $\left(B_{m}\right)$ since the $m$-regularity of $X$ implies that the homogeneous ideal of $X$ is generated in degree $\leq m$ ([16], Lecture 14, Proposition). Also it is clear that $\left(B_{m}\right)$ implies $\left(A_{m}\right)$. On the other hand, the condition $\left(C_{m}\right)$ for $m=d-e+1$ is the famous conjecture on Castelnuovo-Mumford regularity, and sometimes the implication $\left(A_{m}\right) \Rightarrow\left(C_{m}\right)$ for $m$ close to $d-e$, with few trivial exceptions, is also included in the conjecture (see [6]; 9], §4). The conjecture is true for $n=1$ (9], Theorems 1.1 and 3.1), and the first part of it is true for a smooth surface $X$ of $\operatorname{char}(\mathbb{k})=0$ ([15]). For $n \geq 3$, there are nice approaches to the conjecture but it is still open (see [5] and [13] for information). As evidence of the regularity conjecture, it is natural to expect $\left(A_{m}\right)$ and $\left(B_{m}\right)$ for $m=d-e+1$.

Received by the editors October 15, 2007.

2010 Mathematics Subject Classification. Primary 14N05, 14 N15.

Key words and phrases. Secant line, projection, hypersurface, defining equation.

This work was partially supported by Grant-in-Aid for Scientific Research, Japan Society for the Promotion of Science.

(C)2010 American Mathematical Society Reverts to public domain 28 years from publication 
One of the key ideas to study $\left(A_{m}\right)$ and $\left(B_{m}\right)$ for $m=d-e+1$ is based on 17, Theorem 1, where it was shown that $X=\mathrm{E}_{d}(X)$ as a set, and $X=\mathrm{E}_{d}(X)$ as a scheme if $X$ is smooth. To obtain these results, Mumford considered the image $\pi_{\Lambda}(X)$ by the linear projection $\pi_{\Lambda}: \mathbb{P}^{N} \backslash \Lambda \rightarrow \mathbb{P}^{n+1}$ from a general $(e-2)$-plane $\Lambda \subseteq \mathbb{P}^{N}$ and observed that the pull-back of the hypersurface $\pi_{\Lambda}(X)$ separates a point $v \in \mathbb{P}^{N} \backslash X$ from $X$ if $\Lambda$ is suitably chosen according to $v$. In this paper, we will also consider linear projections $\pi_{\Lambda}$ with $\Lambda \cap X \neq \emptyset$.

Now we will state our results. First we deal with $\left(A_{d-e+1}\right)$. We say that a line $L$ is secant to $X$ if $X \cap L$ is finite of length $>0$. We say that a secant line $L$ to $X$ is standard if $\operatorname{dim} \pi_{L}(X \backslash L)=\operatorname{dim} X$ for the linear projection $\pi_{L}$ of $\mathbb{P}^{N} \backslash L$ to $\mathbb{P}^{N-2}$ with center $L$.

Theorem 1 (char $\mathbb{k} \geq 0)$. For a standard secant line $L$ to $X$, we have $l(X \cap L) \leq$ $d-e+1$.

For a nonstandard secant line, the same result is expected. But at this stage, no proof about it would be known for char $\mathbb{k} \geq 0$. The result here is a generalization of results in char $\mathbb{k}=0,[2], 3$, 14], and [18. When $X$ is smooth, we have a sharp bound including another invariant of $X([19])$.

Next we deal with $\left(B_{d-e+1}\right)$. To state our result, we introduce some notation:

$$
\begin{aligned}
& \mathrm{B}(X):=\left\{v \in \mathbb{P}^{N} \backslash X \mid l(X \cap\langle v, x\rangle) \geq 2 \text { for general } x \in X\right\}, \\
& \mathrm{C}(X):=\{u \in X \backslash \operatorname{Sing} X \mid l(X \cap\langle u, x\rangle) \geq 3 \text { for general } x \in X\},
\end{aligned}
$$

where Sing $X$ denotes the singular locus of $X$. In other words, these are the loci of points from which $X$ is projected nonbirationally onto its image: in the former, points off $X$, and in the latter, points on $X \backslash \operatorname{Sing} X$. When $e=1$, it is clear that $\left(B_{d}\right)$ holds and $\mathrm{B}(X)=\mathbb{P}^{N} \backslash X$ and $\mathrm{C}(X)=X \backslash \operatorname{Sing} X$ if $d \geq 3$. Thus we consider $\left(B_{d-e+1}\right)$ for $e \geq 2$.

Theorem 2 (char $\mathbb{k}=0)$. Assume $e \geq 2$.

(1) (Calabri and Ciliberto (4, Corollary 2); and Sommese, Verschelde, and Wampler (22])) As sets, $X \subseteq \mathrm{E}_{d-e+1}(X) \subseteq X \cup \mathrm{B}(X)$.

(2) As schemes, $X$ and $\mathrm{E}_{d-e+1}(X)$ are equal outside $\mathrm{B}(X), \mathrm{C}(X)$, and $\operatorname{Sing} X$.

As an application of Theorem 2, we have another proof of Theorem 1 in $\operatorname{char}(\mathbb{k})=$ 0 , since a standard secant line $L$ to $X$ is not contained in $\mathrm{B}(X)$ (see Remark 3.6). As another application, we will prove $\left(B_{d-e+1}\right)$ for a special case:

Corollary 3 (char $\mathbb{k}=0)$. Suppose that $X\left(\subseteq \mathbb{P}^{N}\right)$ with $e \geq 2$ is contained in the image $v_{l}\left(\mathbb{P}^{m}\right) \subseteq \mathbb{P}^{M}\left(M=\left(\begin{array}{c}m+l \\ l\end{array}\right)-1\right)$ of an lth $(l \geq 2)$ Veronese embedding $v_{l}$ of a projective space $\mathbb{P}^{m}$ in $\mathbb{P}^{M}\left(\supseteq \mathbb{P}^{N}\right)$ for some $m>0$. Then $\mathrm{B}(X)$ and $\mathrm{C}(X)$ are empty. Consequently $X=\mathrm{E}_{d-e+1}(X)$ as sets; and $X=\mathrm{E}_{d-e+1}(X)$ as schemes if $X$ is smooth.

Finally we will study the structure of $\mathrm{B}(X)$ and $\mathrm{C}(X)$. We show that $\mathrm{B}(X)$ is a closed subset of $\mathbb{P}^{N} \backslash X$ and that $\mathrm{C}(X)$ is a closed subset of $X \backslash \operatorname{Sing} X$ in (4.1) and (4.2). The set $\mathrm{B}(X)$ was first studied by Beniamino Segre [20] and 21], and later by Calabri and Ciliberto [4. Segre 20] proved that the closure of $\mathrm{B}(X)$ is the union of a finite number of linear subspaces of dimension at most $n-1$ (see Theorem 4.3). Based on Segre's result, we say more about $\mathrm{B}(X)$ and $\mathrm{C}(X)$. Conventionally, we mean $\operatorname{dim} \emptyset=-1$. 
Theorem $4(\operatorname{char} \mathbb{k}=0)$. If $e \geq 2$, then $\operatorname{dim} \mathrm{B}(X) \leq \min \{n-1, \operatorname{dim} \operatorname{Sing} X+1\}$. In particular, if $X$ is smooth (i.e., $\operatorname{dim} \operatorname{Sing} X=-1$ ) and $e \geq 2$, then $\mathrm{B}(X)$ is a finite set.

Theorem 5 (char $\mathbb{k}=0)$. Assume $e \geq 2$. Let $Z$ be an irreducible component of $\mathrm{C}(X)$. Then the closure of $Z$ is a linear subspace of dimension $l \leq \min \{n-$ $1, \operatorname{dim} \operatorname{Sing} X+2\}$.

As a consequence of Theorems 2, 3, 4 and 5, we have the following.

Corollary 6 (char $\mathbb{k}=0$ ). Suppose that $X$ is smooth, $n \geq 2$ and $e \geq 2$. Then $\mathrm{B}(X)$ is a finite set and $\mathrm{C}(X)$ is the union of a finite number of linear subspaces of dimension $\leq 1$. Consequently the intersection of all hypersurfaces containing $X$, of degree $\leq d-e+1$, is equal to $X$ as a scheme, except for a finite union of linear subspaces of dimension $\leq 1$.

Moreover we will show that the inequality in Theorem 5 is sharp by giving an example in (4.10). Also, in Theorem 4.11, we study the singular locus of $X$ contained in the boundary of $\mathrm{C}(X)$.

We organize this paper as follows. In $\S 1$, we summarize some results of inner projections which we will use later. In $\S 2$, we prove Theorem 1 . In $\S 3$, we prove Theorem 2 and Corollary 3. In $\S 4$, we look at the structure of $\mathrm{B}(X)$ and $\mathrm{C}(X)$ and prove Theorems 4.4, which is a strong form of Theorem 4, and Theorem 5.

The author would like to thank the organizers of the conference at KAIST in January 2006, especially Professor Sijong Kwak, for their hospitality. Also the author would like to thank Professor Fyodor Zak for information regarding the results of Segre and Calabri-Ciliberto.

Notation. We use standard terminology from algebraic geometry, e.g, [11. By a point, we always mean a closed point. A general point means a point off a finite union of suitable proper closed subvarieties. For a point $x$ of $X$, by $T_{x}(X)$, we denote the embedded tangent space to $X$ at $x$ in $\mathbb{P}^{N}$. By $\langle Y, Z\rangle$, we denote the linear span of subschemes $Y$ and $Z$ of $\mathbb{P}^{N}$, the smallest linear subspace containing both $Y$ and $Z$. By $\operatorname{Sing} X$ (resp. Sm $X$ ), we denote the singular locus (resp. smooth locus) of $X$.

\section{$\S 1$. INNER PROJECTION}

In this section, we summarize some results of inner projections.

1.1. Let $X \subseteq \mathbb{P}^{N}$ be as in $\S 0$. Let $\Lambda \subseteq \mathbb{P}^{N}$ be a linear subspace of dimension $l$ with $\Lambda \cap X \neq \emptyset$ but $\Lambda \nsupseteq X$. The linear projection $\pi_{\Lambda}: \mathbb{P}^{N} \backslash \Lambda \rightarrow \mathbb{P}^{N-l-1}$ from $\Lambda$ induces a morphism $\pi_{\Lambda, X}: X \backslash \Lambda \rightarrow \mathbb{P}^{N-l-1}$. Let $V$ be the linear space of linear forms on $\mathbb{P}^{N}$, and let $W \subseteq V$ be the subspace of linear forms vanishing on $\Lambda$, i.e., $\mathbb{P}^{N}=\mathbb{P}(V)$ and $\mathbb{P}^{N-l-1}=\mathbb{P}(W)$. Then $\pi_{\Lambda, X}$ is defined by the surjection $\varepsilon: W \otimes \mathcal{O}_{X} \rightarrow \mathcal{I}_{X \cap \Lambda / X} \otimes \mathcal{O}_{X}(1)$ induced from $V \otimes \mathcal{O}_{X} \rightarrow \mathcal{O}_{X}(1)$. We can identify $\mathbb{P}^{N-l-1}$ to be a subspace of $\mathbb{P}^{N}$ disjoint from $\Lambda$. Let $\sigma: \hat{X} \rightarrow X$ be the blowing up of $X$ by the ideal sheaf $\mathcal{I}_{X \cap \Lambda / X}$ of $\mathcal{O}_{X}$, and let $E$ be the exceptional Cartier divisor. Then we have the morphism $\hat{\pi}_{\Lambda, X}: \hat{X} \rightarrow \mathbb{P}^{N-l-1}$ with $\hat{\pi}_{\Lambda, X} \circ \sigma=\pi_{\Lambda, X}$ as a rational map, since $\varepsilon$ induces a surjection $\hat{\varepsilon}: W \otimes \mathcal{O}_{\hat{X}} \rightarrow \mathcal{O}_{\hat{X}}(-E) \otimes \sigma^{*} \mathcal{O}_{X}(1)$. Thus the closure $\bar{X}$ of $\pi_{\Lambda}(X \backslash \Lambda)$ is exactly $\hat{\pi}_{\Lambda, X}(\hat{X})$ and hence

$$
\bar{X}=\pi_{\Lambda}(X \backslash \Lambda) \cup \hat{\pi}_{\Lambda, X}(E), \quad \text { as a set. }
$$


1.1.2. Assume $\operatorname{dim} \bar{X}=\operatorname{dim} X$. Then $Y:=\left\{\bar{x} \in \bar{X} \mid \operatorname{dim} \hat{\pi}_{\Lambda, X}^{-1}(\bar{x}) \geq 1\right\} \cup \hat{\pi}_{\Lambda, X}(E)$ is a proper closed subset of $\bar{X}$. Thus the induced morphism $\hat{X} \backslash \hat{\pi}_{\Lambda, X}^{-1}(Y) \rightarrow \bar{X} \backslash Y$ from $\hat{\pi}_{\Lambda, X}$ is finite, and hence for the open set $U:=\sigma\left(\hat{X} \backslash \hat{\pi}_{\Lambda, X}^{-1}(Y)\right)$ of $X, \pi_{\Lambda, X} \mid U$ is finite.

1.1.3. On the other hand, assume that $e \geq 2$ and $\Lambda=\{z\}$ for a smooth point $z$ of $X$. Then (1) $\operatorname{dim} \bar{X}=\operatorname{dim} X$, since $X$ is not a cone with vertex $z$ nor linear; (2) $\hat{\pi}_{z, X}(E)=\pi_{z}\left(T_{z}(X) \backslash\{z\}\right)$, since the map $H^{0}\left(\hat{\varepsilon} \otimes \mathcal{O}_{E}\right)$ is $\varepsilon \otimes \mathbb{k}(z): W \rightarrow m_{z} / m_{z}^{2}$ for the maximal ideal $m_{z}$ of $\mathcal{O}_{X, z} ;(3) 0 \leq \operatorname{deg} \bar{X} \leq d-1$ by Bézout's theorem or by computing the degree of $\mathcal{O}_{\hat{X}}(-E) \otimes \sigma^{*} \mathcal{O}_{X}(1)$; (4) For a point $x(\neq z) \in X$, $l(X \cap\langle z, x\rangle)=l\left(\hat{\pi}_{z, X}^{-1}\left(\pi_{z, X}(x)\right)\right)+1$ by a local computation (see for example, [7], p.269, (8.4.3)). In particular, if $l(X \cap\langle z, x\rangle)=2$ for a point $x(\neq z) \in X$, then $\pi_{z, X}$ is an embedding at $x$ by (1.1.2).

1.2. Returning to the original situation in (1.1), we look at the behavior of tangent spaces under the projection. By $\pi: X \backslash \Lambda \rightarrow \bar{X}$ we denote the induced morphism from $\pi_{\Lambda, X}$. Let $x$ be a smooth point of $X$ with $x \notin \Lambda$. Assume $\bar{x}:=\pi_{\Lambda}(x) \in \operatorname{Sm} \bar{X}$. By comparing the bundles of the principal part with respect to $\mathcal{O}_{X}(1)$ and $\mathcal{O}_{\bar{X}}(1)$ (see [12], (IV.A)), we have $\pi_{\Lambda}\left(T_{x}(X) \backslash \Lambda\right) \subseteq T_{\bar{x}}(\bar{X})$, and the equality holds if and only if $\pi$ is smooth at $x$. If the equality holds, then $\left\langle T_{\bar{x}}(\bar{X}), \Lambda\right\rangle=\left\langle T_{x}(X), \Lambda\right\rangle$, and hence

$$
\operatorname{dim} \bar{X}=\operatorname{dim} \pi_{\Lambda}\left(T_{x}(X) \backslash \Lambda\right)=n-\operatorname{dim}\left(T_{x}(X) \cap \Lambda\right)-1
$$

and

$$
T_{x^{\prime}}(X) \subseteq\left\langle T_{x}(X), \Lambda\right\rangle \quad \text { for each } \quad x^{\prime} \in X_{\bar{x}} \cap \operatorname{Sm} X,
$$

where $X_{\bar{x}}$ is the closure of $\pi_{\Lambda, X}(\bar{x})$. In particular, if $\operatorname{char}(\mathbb{k})=0$, by the generic smoothness of $\left.\pi\right|_{\operatorname{Sm} X \backslash \Lambda}$ (e.g. [11], III.10.7), the above holds for general $x \in \operatorname{Sm} X$ and its image $\bar{x}=\pi_{\Lambda}(x)$.

\section{§2. Multisecant lines: Proof of Theorem 1}

In this section we will prove Theorem 1. The key is to find a hypersurface $F$ of degree $\leq d$, containing $X$, and meeting $L$ in at least $e-1$ distinct points off $X \cap L$.

2.1. Proof of Theorem 1. First we claim that if $M \subseteq \mathbb{P}^{N}$ is a general $e$ dimensional linear subspace containing $L$, then $M \cap X$ is finite and containing at least $e-1$ distinct points off $L$. To prove this, consider the linear projection $\pi_{L}: \mathbb{P}^{N} \backslash L \rightarrow \mathbb{P}^{N-2}$ from $L$. The closure $\bar{X}$ of $\pi_{L}(X \backslash L)$ has dimension $n$ by assumption. Since $X$ is nondegenerate, then so is $\bar{X} \subseteq \mathbb{P}^{N-2}$. Thus $\bar{d}:=\operatorname{deg} \bar{X} \geq$ $e-1$ (see for example [10], (18.12); 8], (I.4.2)). Since $\bar{M}:=\pi_{L}(M \backslash L)$ is a general $(e-2)$-dimensional linear subspace of $\mathbb{P}^{N-2}$, by Bézout's and Bertini's Theorems, $\bar{X} \cap \bar{M}$ is $\bar{d}$ distinct points, contained in a nonempty open subset $U$ of $\bar{X}$ over which the induced morphism $\pi_{L, X}$ is a finite morphism (see (1.1.2) for $U$ ). This implies the claim.

Let $M \subseteq \mathbb{P}^{N}$ be a general $e$-dimensional linear subspace containing $L$. By the first part, as sets, $(M \backslash L) \cap X=\left\{x_{1}, \ldots, x_{m}\right\}$ and $L \cap X=\left\{y_{1}, \ldots, y_{t}\right\}$ for some distinct points $x_{j}$ and $y_{i}$ with

$$
m(\geq \bar{d}) \geq e-1 .
$$


Take a general $(e-2)$-dimensional linear subspace $\Lambda \subseteq M$. We may assume that $\Lambda$ is disjoint from $X, L$, and lines $\left\langle y_{i}, x_{j}\right\rangle$ and $\left\langle x_{j}, x_{k}\right\rangle(i=1, \ldots, t ; j \neq k=1, \ldots, m)$. Consequently, $\left\langle\Lambda, x_{j}\right\rangle \cap L$ is a point, say $z_{j}$, and $\left\{z_{j}\right\}$ are $m$ distinct points off $X \cap L$. Now consider the linear projection $\pi_{\Lambda}: \mathbb{P}^{N} \backslash \Lambda \rightarrow \mathbb{P}^{n+1}$ from $\Lambda$. The image $X^{\prime}:=\pi_{\Lambda}(X) \subseteq \mathbb{P}^{n+1}$ is a hypersurface of degree $d^{\prime}(\leq d)$, since the projection $\pi_{\Lambda, X}: X \rightarrow \mathbb{P}^{n+1}$ is finite. Let $F$ be the hypersurface obtained by the pullingback of $X^{\prime} \subseteq \mathbb{P}^{n+1}$ by $\pi_{\Lambda}$. In other words, $F$ is the cone over $X^{\prime}$ with vertex $\Lambda$ if we consider $\mathbb{P}^{n+1}$ to be a subspace of $\mathbb{P}^{N}$, disjoint from $\Lambda$. Thus $\operatorname{deg} F=d^{\prime} \leq d, F \supseteq X$ and $F \cap L \supseteq(X \cap L) \cup\left\{z_{1}, \ldots, z_{m}\right\}$. Moreover $F \nsupseteq L$, since the closure of $\pi_{\Lambda}^{-1}\left(\pi_{\Lambda}(L)\right)$ is $\langle L, \Lambda\rangle=M$ and $M \cap X$ is finite. Consequently $d^{\prime}=l(F \cap L) \geq l(X \cap L)+m$, and hence

$$
l(X \cap L) \leq d^{\prime}-m \leq d-m \leq d-e+1 .
$$

Remark 2.2. Under the same notation and assumptions as in Theorem 1, if $l(X \cap L)=d-e+1$, then the closure $\bar{X}$ of $\pi_{L}(X \backslash L)$ is a variety of minimal degree, i.e., $\bar{d}=\bar{e}+1$ (see [10, (19.9); 8], (I.2.2), (I.5.10)). Furthermore, if $\operatorname{char}(\mathbb{k})=0$, then $\pi: X \backslash L \rightarrow \bar{X}$ is separable and hence $\bar{X}$ is birational to $X$. Indeed, if $l(X \cap L)=d-e+1$, then it follows from (2.1.2) that $m=e-1$ and $d^{\prime}=d$. Hence $m=\bar{d}=e-1$ by (2.1.1), as required.

Remark 2.3. If a secant line $L$ meets $X$ only at $\operatorname{Sm} X$ and $e \geq 2$, by a Bertinitype theorem, $X \cap H$ is irreducible and reduced for a general hyperplane $H \supseteq L$, and hence $L$ is standard (see [19, Lemma for the proof; see also [14] and [18, Lemma 2.1). On the other hand, if $X$ has bad singularity at $X \cap L$, for a general hyperplane $H \supseteq L$ of $\mathbb{P}^{N}, X \cap H$ is not necessarily irreducible (see [18, Example 2.2(2)). Moreover, if $X$ is integral and regular in codimension 1, but not normal at $x \in X \cap L$, then $X$ has Serre's condition $S_{1}$ but not $S_{2}$ at $x$ (see [1, VII (2.2) and (2.13)), and hence $X \cap H$ is not $S_{1}$ at $x$, i.e., $X \cap H$ is not reduced. Thus to obtain Theorem 1, the method of taking hyperplane sections used in [2], 14], and [19] does not work in case $X \cap L \nsubseteq \operatorname{Sm} X$.

\section{§3. Hypersurfaces containing projective variety: Proof of TheOrem 2}

Let $X \subseteq \mathbb{P}^{N}$ be as in $\S 0$. In this section, we always assume $\operatorname{char}(\mathbb{k})=0$ and will prove Theorem 2. This assumption is necessary only to apply the trisecant lemma, which asserts for general points $x, y$ of $X, l(X \cap\langle x, y\rangle)=2$ if $e \geq 2$ and $\operatorname{char}(\mathbb{k})=0$ : In fact, the set of points $x \neq y \in X$ with $l(X \cap\langle x, y\rangle) \geq 3$ is a closed subset of $X \times X \backslash \Delta_{X}$, and also a proper subset by the trisecant lemma (see for example [1], IV 3.8) for curves which are obtained by hyperplane sections of $X$.

Theorem 2 is reduced to proving Propositions 3.1 and 3.2. For scheme-theoretic part (2), see [17], p.34, Lemma.

Proposition 3.1 (char $\mathbb{k}=0$ ). Let $v$ be a point of $\mathbb{P}^{N} \backslash X$. Suppose $v \notin \mathrm{B}(X)$ if $e \geq 2$. Then there exists a hypersurface $F$ of degree $\leq d-e+1$ such that $F \supseteq X$ but $v \notin F$.

Proof. When $e=1$, this is clear. By induction on $e$, suppose $e \geq 2$. Let $x$ be a general point of $X$, so that $x \in \operatorname{Sm} X$ and $l(X \cap\langle v, x\rangle)=1$ by $v \notin \mathrm{B}(X)$. Consider the projection $\pi_{x}: \mathbb{P}^{N} \backslash\{x\} \rightarrow \mathbb{P}^{N-1}$ from $x$ and let $\bar{X}$ be the closure of $\pi_{x}(X \backslash\{x\})$. By (1.1.1) and (1.1.3), $\bar{X}$ is a nondegenerate projective variety of degree $\bar{d} \leq d-1$, 
and codimension $\bar{e}=e-1$, and $\bar{X}=\pi_{x}(X \backslash\{x\}) \cup \pi_{x}\left(T_{x}(X) \backslash\{x\}\right)$. Thus $\bar{v}:=\pi_{x}(v) \notin \bar{X}$, since $l(X \cap\langle v, x\rangle)=1$. We assume, for a moment, that $\bar{v} \notin \mathrm{B}(\bar{X})$ for $\bar{e}=e-1 \geq 2$, and will complete the induction. By the induction, we have a hypersurface $\overline{\bar{F}} \subseteq \mathbb{P}^{N-1}$, of degree $\leq \bar{d}-\bar{e}+1(\leq d-e+1)$ such that $\bar{F} \supseteq \bar{X}$ but $\bar{v} \notin \bar{F}$. Let $F$ be the hypersurface obtained by the pulling-back of $\bar{F}$ by $\pi_{x}$. Then $\operatorname{deg} F=\operatorname{deg} \bar{F} \leq d-e+1, F \supseteq X$ and $v \notin F$, since $F$ is the cone over $\bar{F}$ with vertex $x$, as required.

To conclude the proof, we will show that $\bar{v} \notin \mathrm{B}(\bar{X})$ for $e \geq 3$. By contradiction, we assume that for a general point $y \in X$ with $\bar{y}:=\pi_{x}(y) \in \operatorname{Sm} \bar{X}$,

$$
l(\langle\bar{v}, \bar{y}\rangle \cap \bar{X}) \geq 2 .
$$

By the generality of $x$ and $y$ and the trisecant lemma, the projection $\pi_{x, X}: X \backslash$ $\{x\} \rightarrow \mathbb{P}^{N-1}$ is an embedding at $y$ (see $\left.(1.1 .3)(4)\right)$, and consequently $T_{\bar{y}}(\bar{X})=$ $\pi_{x}\left(T_{y}(X)\right)$ (see (1.2)). Moreover, $v \notin T_{x}(X), v \notin T_{y}(X), x \notin\left\langle T_{y}(X), v\right\rangle$, and $y \notin\left\langle T_{x}(X), v\right\rangle$ since $v \notin \mathrm{B}(X)$ and $x$ and $y$ are general in nondegenerate $X$, and consequently

$$
\bar{v} \notin T_{\bar{y}}(\bar{X})=\pi_{x}\left(T_{y}(X)\right) \quad \text { and } \quad\langle\bar{y}, \bar{v}\rangle \cap \pi_{x}\left(T_{x}(X) \backslash\{x\}\right)=\emptyset .
$$

By (3.1.1) and (3.1.2), there is a point $\bar{z} \in\langle\bar{v}, \bar{y}\rangle \cap \bar{X}$ distinct from $\bar{y}$ which is an image $\pi_{x}(z)$ of some $z \in X$. This means that $\langle v, x, y\rangle$ contains $z \in X$ off $\langle v, x\rangle$ and $\langle v, y\rangle$, since $x$ and $y$ are general and $v \notin \mathrm{B}(X)$. Thus for the linear projection $\pi_{v}: \mathbb{P}^{N} \backslash\{v\} \rightarrow \mathbb{P}^{N-1}$ from $v$, the image $\pi_{v}(X)$ has a secant line meeting at the three distinct points $\pi_{v}(x), \pi_{v}(y)$ and $\pi_{v}(z)$. This contradicts the trisecant lemma, since $\pi_{v}(x)$ and $\pi_{v}(y)$ are general points of $\pi_{v}(X)$ because of the generality of $x$ and $y$ in $X$.

Proposition 3.2 (char $\mathbb{k}=0)$. Let $u$ be a smooth point of $X$ with embedded tangent space $T_{u}(X) \subseteq \mathbb{P}^{N}$ and let $w$ be a point of $\mathbb{P}^{N} \backslash T_{u}(X)$. Suppose $u \notin \mathrm{C}(X)$ if $e \geq 2$. Then there exists a hypersurface $F$ containing $X$, of degree $\leq d-e+1$, such that $F$ is smooth at $u$ with $w \notin T_{u}(F) \subseteq \mathbb{P}^{N}$.

Proof. When $e=1$, this is clear. By induction, suppose $e \geq 2$. Let $x$ be a general point of $X$. Then $x \in \operatorname{Sm} X$ with $l(X \cap\langle x, u\rangle)=2$ and $x \notin\left\langle T_{u}(X), w\right\rangle$. Consider the projection $\pi_{x}: \mathbb{P}^{N} \backslash\{x\} \rightarrow \mathbb{P}^{N-1}$ from $x$, and let $\bar{X}$ be the closure of $\pi_{x}(X \backslash\{x\})$. The projection $\pi_{x, X}: X \backslash\{x\} \rightarrow \mathbb{P}^{N-1}$ is an embedding at $u$ (see (1.1.3)), and hence $\bar{u}:=\pi_{x}(u) \in \operatorname{Sm} \bar{X}$, and $\bar{w}:=\pi_{x}(w) \notin T_{\bar{u}}(\bar{X})=\pi_{x}\left(T_{u}(X)\right)$ (see $\left.(1.2)\right)$. Moreover $\bar{X}$ is a nondegenerate projective variety of degree $\bar{d} \leq d-1$, dimension $\bar{n}=n$, and codimension $\bar{e}=e-1$, with $\bar{X}=\pi_{x}(X \backslash\{x\}) \cup \pi_{x}\left(T_{x}(X) \backslash\{x\}\right.$ ) (see (1.1.1) and (1.1.3)). We assume, for a moment, that $\bar{u} \notin \mathrm{C}(\bar{X})$ for $e \geq 3$, and will complete the induction. By induction, we have a hypersurface $\bar{F} \subseteq \mathbb{P}^{n+e-1}$ containing $\bar{X}$, of degree $\leq \bar{d}-\bar{e}+1(\leq d-e+1)$, smooth at $\bar{u}$ with $\bar{w} \notin T_{\bar{u}}(\bar{F})$. The hypersurfaces $F$ obtained by the pulling-back of $\bar{F}$ by $\pi_{x}$ is of degree $\leq \bar{d}-\bar{e}+1$, smooth at $u$ with $w \notin T_{u}(F)$, as required.

To conclude our proof, we will show that $\bar{u} \notin \mathrm{C}(\bar{X})$ for $e \geq 3$. By contradiction, assume that for general $y \in X$ with $\bar{y}:=\pi_{x}(y) \in \operatorname{Sm} \bar{X}, \bar{l}(\langle\bar{y}, \bar{u}\rangle \cap \bar{X}) \geq 3$. By the generality of $x$ and $y$ and the trisecant lemma, $\pi_{x, X}$ is an embedding at $y$, and hence $T_{\bar{y}}(\bar{X})=\pi_{x}\left(T_{y}(X)\right)$. Moreover, $y \notin\left\langle T_{u}(X), x\right\rangle, y \notin\left\langle T_{x}(X), u\right\rangle$, and $x \notin\left\langle T_{y}(X), u\right\rangle$. Hence $\bar{y} \notin T_{\bar{u}}(\bar{X}), \bar{y} \notin\left\langle\bar{u}, \pi_{x}\left(T_{x}(X) \backslash\{x\}\right)\right\rangle$, and $\bar{u} \notin T_{\bar{y}}(\bar{X})$. Consequently there is a point $\bar{z} \in \bar{X} \cap\langle\bar{y}, \bar{u}\rangle$ distinct from $\bar{y}$ and $\bar{u}$ with $\bar{z}=\pi_{x}(z)$ for some $z \in X$. This means that $z$ lies on $\langle u, x, y\rangle$, but off $\langle u, x\rangle$ and $\langle u, y\rangle$ by 
$u \notin \mathrm{C}(X)$. Thus for the projection $\pi_{u}: \mathbb{P}^{N} \backslash\{u\} \rightarrow \mathbb{P}^{N-1}$ from $u$, the closure $X^{\prime}$ of $\pi_{u}(X \backslash\{u\})$ has a general secant line $\left\langle\pi_{u}(x), \pi_{u}(y)\right\rangle$ meeting at the three distinct points, which contradicts the trisecant lemma.

Remark 3.3. If $v \in \mathrm{B}(X)$, then $\pi_{x}(v) \in \pi_{x}(X \backslash\{x\})$ for any $x \in X$. Thus the points of $\mathrm{B}(X)$ cannot separate from $X$ by the hypersurfaces obtained in (3.1). On the other hand, if $u \in \mathrm{C}(X)$, then $\pi_{x}(u) \in \operatorname{Sing} \pi_{x}(X \backslash\{x\})$ for any $x \in X$. Thus at the points of $\mathrm{C}(X)$, the hypersurfaces in (3.2) cannot separate the tangent space of $X$ from $\mathbb{P}^{N}$.

Example 3.4. Let $X \subseteq \mathbb{P}^{N}(N=n+e)$ be a projective variety of dimension $n$, degree $d$, and codimension $e$ over an algebraically closed field $\mathbb{k}$ of char $\mathbb{k}=0$. Assume $X$ is a variety of minimal degree, or of delta genus $\Delta(X)=0$, i.e., $d=e+1$ (see [10, (19.9); 8], (I.2.2), (I.5.10)). Assume $e \geq 2$, or equivalently $d \geq 3$. Then $\mathrm{B}(X)=\emptyset$. Moreover, $X$ has no 3 -secant line and hence $\mathrm{C}(X)=\emptyset$.

Proof. Suppose $\mathrm{B}(X) \neq \emptyset$, and we will show that $e=1$. Consider the projection $\pi_{v}: \mathbb{P}^{N} \backslash\{v\} \rightarrow \mathbb{P}^{N-1}$ from a point $v \in \mathrm{B}(X)$. Set $\bar{X}=\pi_{v}(X)$ and $\bar{d}=\operatorname{deg} \bar{X}$. Then $\bar{d} \leq d / 2(=(e+1) / 2)$. Since $\bar{X}$ is nondegenerate in $\mathbb{P}^{N-1}$, we have $\bar{d} \geq$ $N-n=e$ by [10], (18.12) or [8],(I.4.2). From these two inequalities, we obtain $e \leq 1$, and hence $e=1$, as required. The second part follows from the first part and Theorem 2, noting that $d-e+1=2$. (Or directly, if there is a 3 -secant line $L$, consider the projection from $L$, and follow the same argument as above. Then we have a contradiction $N=n$.)

3.5. Proof of Corollary 3. We have only to show that $\mathrm{B}(X)$ and $\mathrm{C}(X)$ are empty. First we will prove $\mathrm{B}(X)=\emptyset$. By contradiction, assume there is a point $v \in \mathrm{B}(X)$. For general $x_{1} \neq x_{2} \in X$, consider the lines in $\mathbb{P}^{M}$ joining $x_{i}$ and $v$, and observe on the lines that there exist points $y_{i} \in X$ different from $x_{i}$. Let $L_{i}$ $(i=1,2)$ be the line in $\mathbb{P}^{m}$ joining the preimages of $x_{i}$ and $y_{i}$ in $\mathbb{P}^{m}$. Note that $v \in\left\langle v_{l}\left(L_{i}\right)\right\rangle$. If $L_{1}=L_{2}$, then $X=v_{l}\left(L_{1}\right)$ with $\mathrm{B}(X) \neq \emptyset$, and hence $X$ is conic by Example 3.4, a contradiction. Thus $L_{1} \neq L_{2}$. On the other hand, it is easy to see for two distinct lines $\ell_{1}, \ell_{2}$ in $\mathbb{P}^{m}$ that

(1) $\left\langle v_{l}\left(\ell_{1}\right)\right\rangle \cap\left\langle v_{l}\left(\ell_{2}\right)\right\rangle=\emptyset$ if $\ell_{1}$ and $\ell_{2}$ are disjoint, and that

(2) $\left\langle v_{l}\left(\ell_{1}\right)\right\rangle \cap\left\langle v_{l}\left(\ell_{2}\right)\right\rangle=v_{l}\left(\ell_{1} \cap \ell_{2}\right)$ if $\ell_{1} \cap \ell_{2} \neq \emptyset$.

Therefore $v \in v_{l}\left(L_{1} \cap L_{2}\right)$. This implies that $v_{l}\left(L_{1}\right)$ has a 3 -secant line $\left\langle v, x_{i}\right\rangle=$ $\left\langle x_{i}, y_{i}\right\rangle$, which contradicts Example 3.3, as required. The second part $\mathrm{C}(X)=\emptyset$ follows from the same argument above for $u \in \mathrm{C}(X)$ instead of $v \in \mathrm{B}(X)$.

Remark 3.6. Proposition 3.1 implies Theorem 1 in case char $\mathbb{k}=0$ : Indeed, since $L$ is standard, $L \backslash X \nsubseteq \mathrm{B}(X)$ by the argument as in the proof of (4.4). By (3.1), we obtain a hypersurface $F$ containing $X$ but not $L$. Consequently $l(X \cap L) \leq$ $l(F \cap L) \leq d-e+1$.

\section{$\S 4$. Study of $\mathrm{B}(X)$ and $\mathrm{C}(X)$ : Proof of Theorems 4 and 5}

Let $X \subseteq \mathbb{P}^{N}$ be as in $\S 0$. In this section, we assume char $\mathbb{k}=0$. We will study the structure of $\mathrm{B}(X)$ and $\mathrm{C}(X)$ in $(0.1)$, and prove Theorems 4 and 5 . As a consequence, we will obtain Corollary 6 .

First we will show that $\mathrm{B}(X)$ and $\mathrm{C}(X)$ are algebraic sets.

Lemma 4.1. $\mathrm{B}(X)$ is a closed subset of $\mathbb{P}^{N} \backslash X$. 
Proof. For a hyperplane $H \subseteq \mathbb{P}^{N}$, we set $U:=\mathbb{P}^{N} \backslash(X \cup H)$. We have only to show that $\mathrm{B}(X) \cap U$ is closed in $U$. To this purpose, consider the morphism $\varpi: U \times X \rightarrow U \times \mathbb{P}^{N-1}$ defined by $(u, x) \mapsto(u,\langle u, x\rangle \cap H)$, that is, the family of the projections $\pi_{u, X}: X \rightarrow \mathbb{P}^{N-1}$ from $u \in U$ to $H \cong \mathbb{P}^{N-1}$. Note that $\varpi$ is projective, since $U \times X \rightarrow U$ is projective and $U \times \mathbb{P}^{N-1} \rightarrow U$ is separated (see [11, Ex.II.4.9). Moreover $\varpi$ is finite, since $\varpi$ is quasi-finite (see [11, Ex.III.11.2). Hence $\mathcal{W}:=\left\{(u, \bar{x}) \in U \times \mathbb{P}^{N-1} \mid \operatorname{dim}_{\mathbb{k}(u, \bar{x})} \varpi_{*} \mathcal{O}_{U \times X} \otimes \mathbb{k}(u, \bar{x}) \geq 2\right\}$ is the set of points $(u, \bar{x}) \in U \times \mathbb{P}^{N-1}$ whose fibre $\varpi^{-1}(u, \bar{x})\left(\cong \pi_{u}^{-1}(\bar{x})\right)$ is of length at least 2 . Moreover $\mathcal{W}$ is closed in $U \times \mathbb{P}^{N-1}$ by [11, Ex.II.5.8, and hence the first projection $p_{1}: \mathcal{W} \rightarrow U$ is projective. Therefore a point $u \in U$ is contained in $\mathrm{B}(X) \cap U$ if and only if the fibre $\mathcal{W}_{u}:=p_{1}^{-1}(u)$ is dense in $\pi_{u}(X)$, i.e., $\mathcal{W}_{u}=\pi_{u}(X)$. Then $\mathcal{W}_{u}=\pi_{u}(X)$ if and only if $\operatorname{dim} \mathcal{W}_{u}=n$, since $X$ is irreducible and $\pi_{u}$ is finite. Thus $\mathrm{B}(X) \cap U=\left\{u \in U \mid \operatorname{dim} \mathcal{W}_{u} \geq n\right\}$, and hence $\mathrm{B}(X) \cap U$ is closed in $U$, by [11, Ex.II.3.22 (d) and the properness of $p_{1}$, as required.

Lemma 4.2. $\mathrm{C}(X)$ is a closed subset of the smooth locus $\operatorname{Sm} X$ of $X$.

Proof. For a hyperplane $H \subseteq \mathbb{P}^{N}$, we have only to show that $\mathrm{C}(X) \backslash H$ is closed in $X_{0}:=\operatorname{Sm} X \backslash H$. Recall that the linear projection $\pi_{z, X}: X \backslash\{z\} \rightarrow \mathbb{P}^{N-1}$ from $z \in X_{0}$ to $H \cong \mathbb{P}^{N-1} \subseteq \mathbb{P}^{N}$ is extendable to the morphism $\hat{\pi}_{z, X}: \hat{X}_{z} \rightarrow \mathbb{P}^{N-1}$ by taking the blowing-up $\sigma_{z}: \hat{X}_{z} \rightarrow X$ of $X$ at $z$ (see (1.1)). To construct the family of $\hat{\pi}_{z, X}$ over $z \in X_{0}$, consider the family of linear projections $\left(\mathbb{P}^{N} \backslash H\right) \times \mathbb{P}^{N} \rightarrow$ $\mathbb{P}^{N-1} \cong H \subseteq \mathbb{P}^{N},(z, x) \mapsto\langle z, x\rangle \cap H$ whose base locus is the diagonal of $\mathbb{P}^{N} \backslash H$. Its restriction $\varpi_{2}: \mathcal{X}:=X_{0} \times X \rightarrow \mathbb{P}^{N-1}$ is a rational map whose base locus is the diagonal $\Delta_{X_{0}}$ of $X_{0}$. By taking the blowing-up $\sigma: \widehat{\mathcal{X}} \rightarrow \mathcal{X}$ by the ideal sheaf of $\Delta_{X_{0}}$ with reduced structure, we have an extension $\hat{\varpi}_{2}: \widehat{\mathcal{X}} \rightarrow \mathbb{P}^{N-1}$ of $\varpi_{2}$. Since $\mathcal{J}_{\Delta_{X_{0}} / \mathcal{X}} \mid\{z\} \times X \cong \mathcal{J}_{\{z\} / X}$ by a local computation, $\sigma$ is the family of blowing-ups $\sigma_{z}$ for $z \in X_{0}$. Thus $\hat{\varpi}:=\left(p_{1} \circ \sigma\right) \times \hat{\varpi}_{2}: \widehat{\mathcal{X}} \rightarrow X_{0} \times \mathbb{P}^{N-1}$ is the required family such that $\hat{\varpi}^{-1}(z, \bar{x})=\hat{\pi}_{z, X}^{-1}(\bar{x})$ for $(z, \bar{x}) \in X_{0} \times \mathbb{P}^{N-1}$. Note that $\hat{\varpi}$ is projective, since $\widehat{\mathcal{X}} \rightarrow X_{0}$ is projective and $X_{0} \times \mathbb{P}^{N-1} \rightarrow X_{0}$ is separated.

To prove $\mathrm{C}(X) \backslash H$ is closed in $X_{0}$, we consider

$$
\mathcal{W}:=\left\{(z, \bar{x}) \in X_{0} \times \mathbb{P}^{N-1} \mid \operatorname{dim}_{\mathbb{k}(z, \bar{x})} \hat{\varpi}_{*} \mathcal{O}_{\hat{\mathcal{X}}} \otimes \mathbb{k}(z, \bar{x}) \geq 2 \text { or } \operatorname{dim} \hat{\pi}_{z, X}^{-1}(\bar{x}) \geq 1\right\} .
$$

By [11, Ex. II.5.8, and Ex. II.3.22 (d), $\mathcal{W}$ is closed in $X_{0} \times \mathbb{P}^{N-1}$, and the first projection $p_{1}: \mathcal{W} \rightarrow X_{0}$ is projective. Moreover $\mathcal{W}$ is the set of points $(z, \bar{x}) \in X_{0} \times \mathbb{P}^{N-1}$ whose fibre $\hat{\varpi}^{-1}(z, \bar{x})\left(\cong \hat{\pi}_{z, X}^{-1}(\bar{x})\right)$ is of length at least 2 , since $\hat{\varpi}$ is finite around $(z, \bar{x})$ if $\operatorname{dim} \hat{\varpi}^{-1}(z, \bar{x})=0$. A point $(z, \bar{x}) \in X_{0} \times \mathbb{P}^{N-1}$ belongs to $\mathcal{W}$ if and only if $l(X \cap\langle z, \bar{x}\rangle) \geq 3$, since $l\left(\hat{\pi}_{z, X}^{-1}(\bar{x})\right) \geq 2$ if and only if $l(X \cap\langle z, \bar{x}\rangle) \geq 3$ (see (1.1.3)). Thus $z \in X_{0}$ belongs to $\mathrm{C}(X) \backslash H$ if and only if the fibre $\mathcal{W}_{z}:=p_{1}^{-1}(z)$ is equal to $\bar{X}$. The last condition is equivalent to $\operatorname{dim} \mathcal{W}_{z} \geq n$. Therefore $\mathrm{C}(X) \backslash H$ is closed in $X_{0}$, as required.

Next we study the structure of $\mathrm{B}(X)$. Recall the following result.

Theorem 4.3 (Beniamino Segre [20]; see also [4]). Let $X \subseteq \mathbb{P}^{N}(N=e+n)$ be a nondegenerate, projective variety of dimension $n$ and codimension $e$. If $e \geq 2$ and $\mathrm{B}(X) \neq \emptyset$, then every irreducible component of the closure of $\mathrm{B}(X)$ is a linear subspace of dimension at most $n-1$. Moreover $\operatorname{dim} \mathrm{B}(X)=n+1$ if and only if $e=1$. 
Based on this result and the idea of the proof, we will prove Theorem 4.4 and Theorem 5. Theorem 4 is an immediate consequence of Theorem 4.4.

Theorem 4.4. Let $X$ be as in $\S 0$. Assume $n \geq 2$ and $e \geq 2$. Let $\Lambda$ be an irreducible component of the closure of $\mathrm{B}(X)$, of dimension $l \geq 0$. Then

(1) $\operatorname{dim} X \cap \Lambda=l-1$, and

(2) $X \cap \Lambda \subseteq \operatorname{Sing} X$.

Consequently $\operatorname{dim} \Lambda \leq \operatorname{dim} \operatorname{Sing} X+1$, and in particular, $\operatorname{dim} \mathrm{B}(X) \leq \operatorname{dim} \operatorname{Sing} X+$ 1.

Proof. When $l=0$, the assertion is trivial, so we assume $l \geq 1$. By (4.3), $\Lambda$ is linear. First we observe the linear projection $\pi_{\Lambda, X}: X \backslash \Lambda \rightarrow \mathbb{P}^{N-l-1}$ from $\Lambda$, in particular, its fibre and image. For a general point $x \in X \backslash \Lambda$, let $X_{\bar{x}}$ be the closure of $\pi_{\Lambda, X}^{-1}(\bar{x})$ over $\bar{x}:=\pi_{\Lambda}(x)$. Then $\operatorname{dim} X_{\bar{x}}=l$. Indeed, $\operatorname{dim} X_{\bar{x}} \geq l$, since a line joining $x$ and general $v \in \Lambda \cap \mathrm{B}(X)$ contains a point of $X$ different from $x$. Hence $\operatorname{dim} X_{\bar{x}}=l$, since $\pi_{\Lambda, X}^{-1}(\bar{x}) \subseteq\langle\Lambda, x\rangle$ and $\Lambda \nsubseteq X$. Consequently the closure $\bar{X}$ of $\pi_{\Lambda, X}(X \backslash \Lambda)$ has dimension $n-l$. Moreover $\bar{d}:=\operatorname{deg} \bar{X} \geq 2$ : If not, $\bar{X}$ is linear and nondegenerate in $\mathbb{P}^{N-l-1}$, and hence $\bar{X}=\mathbb{P}^{N-l-1}$, which implies $e=1$, a contradiction.

Now (1) is clear, since $\operatorname{dim} X_{\bar{x}}=l$ and $X_{\bar{x}} \subseteq\langle\Lambda, x\rangle$ with $\Lambda \nsubseteq X$.

Next we will show (2) in case $n=2$. Then $l=1$ by assumptions $l \geq 1$ and (4.3). Hence $\operatorname{dim} X_{\bar{x}}=1$ and $\bar{X} \subseteq \mathbb{P}^{N-2}$ is a nondegenerate curve of degree $\bar{d} \geq 2$. Let $H$ be a general hyperplane containing $\Lambda$. The first step is to show that

$$
\operatorname{Sing}(X \cap H) \supseteq X \cap \Lambda \text {. }
$$

Since $\bar{H}:=\pi_{\Lambda}(H \backslash \Lambda)$ is a general hyperplane in $\mathbb{P}^{N-2}$, by Bézout's theorem, $\bar{X} \cap \bar{H}$ is $\bar{d}$ distinct points, say $\bar{x}_{1}, \ldots, \bar{x}_{\bar{d}}$, which lie on $\pi_{\Lambda, X}(X \backslash \Lambda)$ (see (1.1.1)). Consequently $X \cap H$ contains $\bar{d}(\geq 2)$ distinct curves $X_{\bar{x}_{i}}$, each of which contains $X \cap \Lambda$ by Lemma 4.5 below. This implies (4.4.1). Now to obtain (2), we assume, to the contrary, that there is a point $z \in \Lambda \cap \operatorname{Sm} X$. Then $H \nsupseteq T_{z}(X)$ by the generality of $H \supseteq \Lambda$ and $\Lambda \neq T_{z}(X)$. Hence $X \cap H$ is smooth at $z$. This contradicts (4.4.1).

We will show (2) in case $n>2$. By contradiction, assume that there is a point $z \in \Lambda \cap \operatorname{Sm} X$. Take a general line $L$ through $z$, contained in $\Lambda$, not contained in $X$, so that $L \cap \mathrm{B}(X)$ is dense open in $L$ by (4.1). Let $H^{\prime}$ be a general hyperplane containing $L$. We claim that the reduced induced structure $X^{\prime}:=\left(X \cap H^{\prime}\right)_{\text {red }}$ is irreducible and nondegenerate in $H^{\prime} \cong \mathbb{P}^{N-1}$ such that $z \in \operatorname{Sm} X^{\prime}$ and the closure of $\mathrm{B}\left(X^{\prime}\right)$ contains $L$. If the claim is proved, by induction on $n$, we have a projective surface $X^{\prime}$ such that the closure of $\mathrm{B}\left(X^{\prime}\right)$ contains $L$ with $L \cap \operatorname{Sm} X^{\prime} \neq \emptyset$, which contradicts the case $n=2$. Thus we have only to show the claim. By the same argument as in the first paragraph, a general fibre of the linear projection $\pi_{L, X}: X \backslash L \rightarrow \mathbb{P}^{N-2}$ has dimension 1 . Hence the image of $\pi_{L, X}$ has dimension $n-1(\geq 2)$. By Bertini's theorem [24], (I.6.3), $X \cap H^{\prime}$ is irreducible and generically reduced. (To be precise, apply Bertini's theorem for the normalization $\tilde{X}$ of $X$ and the pull-back $\tilde{H}^{\prime}$ of $H^{\prime}$, and take the push-forward of $\tilde{X} \cap \tilde{H}^{\prime}$.) Moreover, since $X$ is integral, $(X \backslash L) \cap H^{\prime}$ satisfies Serre's condition $S_{1}$ by [7], (3.4.6), and hence $(X \backslash L) \cap H^{\prime}$ is reduced (see [1], (VII.2.2)). On the other hand, since $X \subseteq \mathbb{P}^{N}$ is nondegenerate, so is $\pi_{L, X}(X \backslash L) \subseteq \mathbb{P}^{N-2}$, and also so is its hyperplane section (see 7], p.116). Consequently $(X \backslash L) \cap H^{\prime}$ is nondegenerate. Moreover, $X \cap H^{\prime}$ 
is smooth at $z \in \Lambda \cap \operatorname{Sm} X$, since $H^{\prime} \nsupseteq T_{z}(X)$ by the generality of $H^{\prime} \supseteq L$ and $T_{z}(X) \neq L$. Finally it is clear that $L \nsubseteq X^{\prime}$ and $L \backslash X^{\prime} \subseteq \mathrm{B}\left(X^{\prime}\right)$. In sum, $X^{\prime}$ satisfies the property we have claimed. This complete the proof.

Lemma 4.5. Let $X \subseteq \mathbb{P}^{N}$ be a nondegenerate projective variety of dimension $n$ and codimension $e \geq 2$. Let $\Lambda \subseteq \mathbb{P}^{N}$ be a linear subspace of dimension $l(1 \leq l<n)$, not contained in $X$. Assume the image of the linear projection $\pi_{\Lambda, X}: X \backslash \Lambda \rightarrow \mathbb{P}^{N-l-1}$ has dimension $n-l$. Then for each $x \in X \backslash \Lambda$, the closure $X_{\bar{x}}$ of the fibre of $\pi_{\Lambda, X}$ over $\bar{x}:=\pi_{\Lambda, X}(x)$ is a hypersurfaces of $\langle\Lambda, x\rangle$, containing $X \cap \Lambda$.

Proof. Let $\bar{X}$ be the closure of $\pi_{\Lambda, X}(X \backslash \Lambda)$ in $\mathbb{P}^{N-l-1}$, and let $\nu: Y \rightarrow \bar{X}$ be a desingularization of $\bar{X}$. Considering the target $\mathbb{P}^{N-l-1}$ of $\pi_{\Lambda, X}$ to be a subspace of $\mathbb{P}^{N}$ disjoint from $\Lambda$, we have a morphism $\bar{X} \rightarrow \mathbb{G}:=\operatorname{Grass}(l+1, N)$ from $\bar{X}$ to the Grassmann of the $(l+1)$-plane in $\mathbb{P}^{N}$, defined by $\bar{x} \mapsto\langle\Lambda, \bar{x}\rangle$. Let $\mathcal{Q}_{Y}$ be the pull-back of the universal quotient bundle $\mathcal{Q}$ on $\mathbb{G}$ by $Y \rightarrow \bar{X} \rightarrow \mathbb{G}$, so that $\mathcal{Q}_{Y} \cong \mathcal{O}_{Y}^{\oplus l+1} \oplus \mathcal{O}_{Y}(1)$, where $\mathcal{O}_{Y}(1):=\nu^{*}\left(\mathcal{O}_{\mathbb{P}^{N-l-1}}(1) \mid \bar{X}\right)$. The projective bundle $\mathbb{P}:=\mathbb{P}_{Y}\left(\mathcal{Q}_{Y}\right)$ with projection $\tau: \mathbb{P} \rightarrow Y$ has a natural morphism $\phi: \mathbb{P} \rightarrow \mathbb{P}^{N}$ defined by the tautological line bundle $\mathcal{O}_{\mathbb{P}}(1)$, which is an embedding except on $\mathbb{P}_{Y}\left(\mathcal{O}_{Y}^{\oplus l+1}\right)$ and the fibres of $\tau$ over the points of $Y$ at which $Y \rightarrow \mathbb{P}^{N-l-1}$ is not an embedding. Thus $\phi(\mathbb{P})$ contains $X$, and $X$ meets an open subset of $\phi(\mathbb{P})$ where $\phi$ is an embedding. Hence we can consider a prime divisor $\tilde{X}$ on a smooth variety $\mathbb{P}$, with a birational, surjective, induced morphism $\tilde{X} \rightarrow X$. Then $\tilde{X}$ is a member of a linear system $\left|\mathcal{O}_{\mathbb{P}}(\mu) \otimes \tau^{*} \mathcal{M}\right|$ for some positive integer $\mu$ and a line bundle $\mathcal{M}$ on $Y$ (see [1], (II.6.11), (II.6.11.1A), (II.6.15), and (Ex.III.12.5)). We write $\mathcal{Q}_{Y}=\mathcal{O}_{Y} z_{0} \oplus \cdots \oplus \mathcal{O}_{Y} z_{l} \oplus \mathcal{O}_{Y}(1) z_{l+1}$ with formal basis $z_{i}$ (or homogeneous coordinates of fibres). Then $\tilde{X}$ is the zero of

$$
G=\sum_{\mu_{0}, \cdots, \mu_{l+1} \geq 0, \mu_{0}+\cdots+\mu_{l+1}=\mu} g_{\mu_{0} \cdots \mu_{l+1}} z_{0}^{\mu_{0}} \cdots z_{l+1}^{\mu_{l+1}} \in H^{0}\left(\mathcal{O}_{\mathbb{P}}(\mu) \otimes \tau^{*} \mathcal{M}\right)
$$

for some $g_{\mu_{0} \cdots \mu_{l+1}} \in H^{0}\left(Y, \mathcal{M} \otimes \mathcal{O}_{Y}\left(\mu_{l+1}\right)\right)$. By $\mathbb{P}_{y}^{l}$ we denote the fiber of $\mathbb{P}_{Y}\left(\mathcal{O}_{Y} z_{0} \oplus \cdots \oplus \mathcal{O}_{Y} z_{l}\right) \rightarrow Y$ over $y \in Y$. Then $\tilde{X} \cap \mathbb{P}_{y}^{l}$ is the subscheme of $\mathbb{P}_{y}^{l}(\subseteq \mathbb{P})$ defined by

$$
\left.G\right|_{\mathbb{P}_{y}^{l}}=\sum_{\mu_{0}, \cdots, \mu_{l} \geq 0, \mu_{0}+\cdots+\mu_{l}=\mu} g_{\mu_{0} \cdots \mu_{l} 0}(y) z_{0}^{\mu_{0}} \cdots z_{l}^{\mu_{l}}
$$

and $\tilde{X} \cap \mathbb{P}_{y}^{l}$ is mapped into $X \cap \Lambda$. If we consider a rational map, defined by $\left\{g_{\mu_{0} \cdots \mu_{l} 0}\right\} \subseteq H^{0}(Y, \mathcal{M})$, from $Y$ to the set $\mathbb{P}^{M}\left(M=\left(\begin{array}{c}l+\mu \\ l\end{array}\right)-1\right)$ of linear forms of degree $\mu$ on $\mathbb{P}^{l}$ with homogeneous coordinates $z_{0}, \ldots, z_{l}$, then this map is a morphism from $Y$ whose image is one point, since $\phi\left(\tilde{X} \cap \mathbb{P}_{y}^{l}\right) \subseteq X \cap \Lambda$ for each $y \in Y$ and since the points of $\mathbb{P}^{M}$ whose zeros, as $\mu$-forms of $\mathbb{P}^{l}=\Lambda$, are contained in $X \cap \Lambda$ are finite. Consequently $\mathcal{M} \cong \mathcal{O}_{Y}$ and $g_{\mu_{0} \cdots \mu_{l} 0} \in H^{0}\left(\mathcal{O}_{Y}\right) \cong \mathbb{k}$, and moreover $g_{\mu_{0} \cdots \mu_{l} 0} \neq 0$ for some $\mu_{0}, \ldots, \mu_{l}$. Therefore set-theoretically, $\phi\left(\tilde{X} \cap \mathbb{P}_{y}^{l}\right)=X \cap \Lambda$ for each $y \in Y$. This implies that the image $\phi\left(\tilde{X} \cap \mathbb{P}\left(\mathcal{Q}_{Y} \otimes \mathbb{k}(y)\right)\right)$ contains $X \cap \Lambda$. For $\left.x \in X \backslash \Lambda, X_{\bar{x}}=\bigcup_{y \in \nu^{-1}(\bar{x})} \phi\left(\tilde{X} \cap \mathbb{P}\left(\mathcal{Q}_{Y} \otimes \mathbb{k}(y)\right)\right)\right)$ as sets. Hence $X_{\bar{x}}$ is a hypersurface, containing $X \cap \Lambda$, as required.

Example 4.6. The induced morphism $\tilde{X} \cap \mathbb{P}_{y}^{l} \rightarrow X \cap \Lambda$ in the proof of Lemma 4.5 is bijective but not necessarily isomorphic. Let $\mathbb{P}$ be the projective bundle $\mathbb{P}_{\mathbb{P}^{1}}(\mathcal{Q})$ over $Y:=\mathbb{P}^{1}$, associated with the vector bundle $\mathcal{Q}=\mathcal{O}_{\mathbb{P}^{1}} z_{0} \oplus \mathcal{O}_{\mathbb{P}^{1}} z_{1} \oplus \mathcal{O}_{\mathbb{P}^{1}}(4) z_{2}$ 
on $\mathbb{P}^{1}$, with projection $\tau: \mathbb{P} \rightarrow \mathbb{P}^{1}$ and the tautological bundle $\mathcal{O}_{\mathbb{P}}(1)$. Here $\left\{z_{i}\right\}$ is a formal basis. Let $s, t$ be the homogeneous coordinates of $\mathbb{P}^{1}$. Let $\phi: \mathbb{P} \rightarrow \mathbb{P}^{5}$ be the morphism defined by $z_{0}, z_{1}, s^{4} z_{2}, s^{3} t z_{2}, s t^{3} z_{2}, t^{4} z_{2} \in H^{0}\left(\mathcal{O}_{\mathbb{P}}(1)\right)$. Let $\tilde{X}_{i}$ $(i=1,2)$ be divisors defined by $G_{i}$ for $G_{1}=z_{1}^{2}-s^{2} t^{2} z_{0} z_{2}$ and $G_{2}=z_{1}^{2}-s^{3} t z_{0} z_{2}$ in $H^{0}\left(\mathcal{O}_{\mathbb{P}}(2)\right)$. Then the images $X_{i}=\phi\left(\tilde{X}_{i}\right)$ are nondegenerate projective surfaces of degree 8. The line $L:=\phi\left(\mathbb{P}\left(\mathcal{O}_{\mathbb{P}^{1}} z_{0} \oplus \mathcal{O}_{\mathbb{P}^{1}} z_{1}\right)\right)$ is contained in the closure of $\mathrm{B}\left(X_{i}\right)$. Moreover $X_{1} \cap L=X_{2} \cap L$ as sets, and $l\left(X_{1} \cap L\right)=4$ and $l\left(X_{2} \cap L\right)=2$. On the other hand, $l\left(\tilde{X}_{1} \cap \mathbb{P}_{y}^{1}\right)=l\left(\tilde{X}_{2} \cap \mathbb{P}_{y}^{1}\right)=2$ for each $y \in \mathbb{P}^{1}$.

Remark 4.7. In Theorem 4.4, to obtain the inequality $\operatorname{dim} \mathrm{B}(X) \leq \operatorname{dim} \operatorname{Sing} X+1$ for $e \geq 2$, there is an easier argument using Bertini's theorem as follows. Assume to the contrary that $l:=\operatorname{dim} \mathrm{B}(X) \geq \operatorname{dim} \operatorname{Sing} X+2$. By (4.3), $l \leq n-1$. For $m=N-l+1$, let $M \subseteq \mathbb{P}^{N}$ be a general $m$-dimensional linear subspace. By Bertini's theorem, $X^{\prime}:=\bar{X} \cap M$ is a smooth projective variety of dimension $n-l+1$, nondegenerate in $M$ (see [10, (18.10) or [7], (3.5.8)). Moreover the closure of $\mathrm{B}\left(X^{\prime}\right)$ contains a line $L$ with $L \nsubseteq X^{\prime}$, since $\mathrm{B}\left(X^{\prime}\right) \supseteq \mathrm{B}(X) \cap M$. Let $M^{\prime} \subseteq M$ be a general $(e+1)$-dimensional linear subspace containing $L$. Since $L \cap X^{\prime} \subseteq \operatorname{Sm} X^{\prime}$, by a Bertini-type theorem (see [14], (2.1); 18], (2.1)), $X^{\prime \prime}:=X^{\prime} \cap M^{\prime}$ is a smooth nondegenerate projective curve in $M^{\prime}$ with $\operatorname{dim} \mathrm{B}\left(X^{\prime \prime}\right) \geq 1$. Since $L \cap \mathrm{B}\left(X^{\prime \prime}\right)$ is dense in $L$ (or apply Theorem 4.3), $X^{\prime \prime}$ lies on the 2-plane spanned by $L$ and a general point $x \in X^{\prime \prime}$, and consequently $e=1$, a contradiction.

4.8. Proof of Theorem 5. For a general (smooth) point $x$ of $X$, let $\Lambda_{x}$ be the linear span $\left\langle Z, T_{x}(X)\right\rangle$. Now, according to Segre [20], we will show that

$$
\operatorname{dim} \Lambda_{x}=n+1
$$

To this purpose, consider the linear projection $\pi_{z, X}: X \backslash\{z\} \rightarrow \mathbb{P}^{N-1}$ from a point $z \in Z$. Since $z \in \operatorname{Sm} X, \pi_{z, X}$ is generically quasi-finite (see (1.1.3)). By the generic smoothness of $\pi_{z, X}$, the line $\langle x, z\rangle$ meets $X$ at a point $y \in \operatorname{Sm} X$ distinct from $x$ and $z$. Moreover $T_{y}(X) \subseteq\left\langle T_{x}(X), z\right\rangle$ (see (1.2.2)). Let $Y$ be an irreducible component of the closure of the set of the points $y \in\langle x, z\rangle \cap X$ for moving $z \in Z$ and fixed $x \in X$. If $y \in Y$ is general, the corresponding point $z$ is also general in $Z$, and hence,

$$
T_{y}(Y) \subseteq T_{y}(X) \subseteq\left\langle T_{x}(X), z\right\rangle \supseteq T_{x}(X)
$$

From this, by considering the projection of $Y$ from $T_{x}(X)$, we observe that $\left\langle T_{x}(X), y\right\rangle$ does not depend on $y \in Y$ (see (1.2.1)). Consequently $\left\langle T_{x}(X), y\right\rangle=\left\langle T_{x}(X), Y\right\rangle=$ $\Lambda_{x}$, which implies (4.8.1).

Let $\Lambda$ be the intersection of $\Lambda_{x}$ for general points $x \in X$, and set $l:=\operatorname{dim} \Lambda$. If $l=n+1$, then $\Lambda=\Lambda_{x}$ and hence $\Lambda$ contains $X$, which contradicts $e \geq 2$. Thus $l \leq n$. Let $\pi_{\Lambda, X}: X \backslash \Lambda \rightarrow \mathbb{P}^{N-l-1}$ be the linear projection from $\Lambda$ to a subspace $\mathbb{P}^{\bar{N}-l-1}$ of $\mathbb{P}^{N}$ disjoint from $\Lambda$, and let $\bar{X}$ be the closure of $\pi_{\Lambda, X}(X \backslash \Lambda)$. For general $x \in X$, let $X_{\bar{x}}$ be the closure of $\pi_{\Lambda, X}^{-1}(\bar{x})$ over $\bar{x}:=\pi_{\Lambda}(x)$. We claim that $l<n$, $\operatorname{dim} X_{\bar{x}}=l$, and

$$
l((X \backslash \Lambda) \cap\langle v, x\rangle) \geq 2
$$

for general $v \in \Lambda$. If $l=n$, i.e., $\operatorname{codim}\left(\Lambda, \Lambda_{x}\right)=1$, then $\operatorname{dim}\left(T_{x}(X) \cap \Lambda\right)=n-1$, and hence $X \subseteq\langle\Lambda, x\rangle$, which contradicts $e \geq 2$. Thus $l<n$. Then $\operatorname{dim}\left(T_{x}(X) \cap\right.$ $\Lambda)=l$ or $l-1$, since $\operatorname{codim}\left(T_{x}(X), \Lambda_{x}\right)=1$. If $\operatorname{dim}\left(T_{x}(X) \cap \Lambda\right)=l$, by $(1.2 .1)$, 
$\operatorname{dim} \bar{X}=n-l-1$, and hence $X$ is the cone over $\bar{X}$ with vertex $\Lambda$, which means $Z \subseteq \Lambda \subseteq \operatorname{Sing} X$, a contradiction. Therefore $\operatorname{dim}\left(T_{x}(X) \cap \Lambda\right)=l-1$. By (1.2.1), $\operatorname{dim} \bar{X}=n-l$, and $\operatorname{dim} X_{\bar{x}}=l$. The fact that $l((X \backslash\{z\}) \cap\langle z, x\rangle) \geq 2$ for $z \in Z$ implies that the $l$-dimensional part of $X_{\bar{x}}$ is a hypersurface of $\langle\Lambda, x\rangle$, of degree $\geq 2$, not containing $\Lambda$. Thus we have (4.8.2).

Next we will show that $\Lambda$ is the closure of $Z$. If $\Lambda \nsubseteq X$, by (4.8.2), a general point of $\Lambda$ lies on $\mathrm{B}(X)$, and hence $Z \subseteq \Lambda \cap X \subseteq \operatorname{Sing} X$ by (4.4), a contradiction. Thus $\Lambda \subseteq X$. Since $Z$ is an irreducible component of $\mathrm{C}(X)$, if $\operatorname{dim} Z<\operatorname{dim} \Lambda$, then $\Lambda \backslash Z \subseteq \operatorname{Sing} X$ by (4.8.2), and hence $Z \subseteq \Lambda \subseteq \operatorname{Sing} X$, a contradiction. Thus $\operatorname{dim} Z=\operatorname{dim} \Lambda$, and hence $\Lambda$ is the closure of $Z$.

Finally we look at $\operatorname{dim} Z$. Note that $\operatorname{dim} Z=\operatorname{dim} \Lambda=\operatorname{dim} X_{\bar{x}}=l$. By (1.2.2), $T_{x^{\prime}}(X) \subseteq\left\langle T_{\bar{x}}(\bar{X}), \Lambda\right\rangle\left(=\left\langle T_{x}(X), \Lambda\right\rangle=\Lambda_{x}\right) \subseteq \mathbb{P}^{N}$ for each $x^{\prime} \in X_{\bar{x}} \cap \operatorname{Sm} X$. Consequently, by the theorem of tangencies $(23],($ I.1.7)),

$$
\begin{aligned}
l=\operatorname{dim} X_{\bar{x}} & \leq \operatorname{dim}\left\langle T_{\bar{x}}(\bar{X}), \Lambda\right\rangle-\operatorname{dim} X+\operatorname{dim}\left(X_{\bar{x}} \cap \operatorname{Sing} X\right)+1 \\
& =2+\operatorname{dim}\left(X_{\bar{x}} \cap \operatorname{Sing} X\right) \leq 2+\operatorname{dim} \operatorname{Sing} X .
\end{aligned}
$$

This completes the proof of Theorem 5 .

4.9. Proof of Corollary 6. If $X$ is smooth, then $\mathrm{B}(X)$ is a finite set and $\mathrm{C}(X)$ is a finite union of linear subspaces of dimension $\leq 1$. The rest follows from Theorem 2 .

Here we will show that the inequality of Theorem 5 is sharp, by giving an example of $X$ whose $\mathrm{C}(X)$ contains a linear subspace of dimension $\operatorname{dim} \operatorname{Sing} X+2$.

Example 4.10. For integers $l \geq 0, n \geq l+2$ and $a_{n} \geq \cdots \geq a_{l+1}>a_{l}=\cdots=a_{0}=$ 0 , let $\mathbb{P}$ be the projective bundle $\mathbb{P}_{\mathbb{P}^{1}}(\mathcal{E})$ over $\mathbb{P}^{1}$, associated with the vector bundle $\mathcal{E}=\bigoplus_{i=0}^{n} \mathcal{O}_{\mathbb{P}^{1}}\left(a_{i}\right)$ on $\mathbb{P}^{1}$, with projection $\tau: \mathbb{P} \rightarrow \mathbb{P}^{1}$ and the tautological bundle $\mathcal{O}_{\mathbb{P}}(1)$. Assume $a_{n} \geq 2$ if $n=l+2$. For a general member $\tilde{X} \in\left|\mathcal{O}_{\mathbb{P}}(\mu) \otimes \tau^{*} \mathcal{O}_{\mathbb{P}^{1}}(1)\right|$ with an integer $\mu \geq 2$, let $X$ be the image of $\tilde{X}$ by the morphism $\phi: \mathbb{P} \rightarrow \mathbb{P}^{N}$ $\left(N=n+1+\sum_{i=0}^{n} a_{i}\right)$ defined by $\left|\mathcal{O}_{\mathbb{P}}(1)\right|$. Let $L \subseteq \mathbb{P}^{N}$ be the $l$-dimensional linear subspace which is the image $\phi(\tilde{L})$ of the subbundle $\tilde{L}=\mathbb{P}_{\mathbb{P}^{1}}\left(\bigoplus_{i=0}^{l} \mathcal{O}_{\mathbb{P}^{1}}\left(a_{i}\right)\right) \subseteq \mathbb{P}$. Then

(1) $L \subseteq X$

(2) Sing $X$ is a subset of $L$, of codimension 2 if $l \geq 1$, and $\operatorname{Sing} X=\emptyset$ if $l=0$;

(3) $\mathrm{C}(X)$ contains $L \backslash \operatorname{Sing} X$.

Hence $\operatorname{dim} \mathrm{C}(X) \geq \operatorname{dim} L=l \geq \operatorname{dim} \operatorname{Sing} X+2$ if $l \geq 1$ and, $\operatorname{dim} \mathrm{C}(X) \geq 0$ and $\operatorname{dim} \operatorname{Sing} X=-1$ if $l=0$. Consequently $\operatorname{dim} \mathrm{C}(X)=\operatorname{dim} \operatorname{Sing} X+2$ for $l \geq 1$.

Proof. Note that $X \backslash L$ is smooth, since $\phi$ gives an embedding of $\mathbb{P} \backslash \tilde{L}$ into $\mathbb{P}^{N}$ and since $\tilde{X}$ is smooth by the generality of $\tilde{X}$. To see (1) and (2), let us look at $\tilde{X} \cap \tilde{L}$ and the induced morphism $\tilde{X} \cap \tilde{L} \rightarrow L$. Let $s, t$ be the homogeneous coordinates of $\mathbb{P}^{1}$ and let $z_{i}$ be the formal basis of $\mathcal{O}_{\mathbb{P}^{1}}\left(a_{i}\right)$ in $\mathcal{E}$. Then $\tilde{X}$ is defined as a subscheme of $\mathbb{P}$ by

$$
G=\sum_{\mu_{0}, \cdots, \mu_{n} \geq 0, \mu_{0}+\cdots+\mu_{n}=\mu} g_{\mu_{0} \cdots \mu_{n}} \cdot z_{0}^{\mu_{0}} \cdots z_{n}^{\mu_{n}} \in H^{0}\left(\mathcal{O}_{\mathbb{P}}(\mu) \otimes \tau^{*} \mathcal{O}_{\mathbb{P}^{1}}(1)\right) \quad\left(\mu_{i} \geq 0\right)
$$

for some homogeneous polynomials $g_{\mu_{0} \cdots \mu_{n}} \in \mathbb{k}[s, t]$ of degree $1+\sum_{i=l+1}^{n} a_{i} \mu_{i}$. Moreover $\tilde{X} \cap \tilde{L}$ is defined by $G \mid \tilde{L}$ in $\tilde{L} \cong \mathbb{P}^{1} \times L$. Since $\tilde{L}$ is defined by $z_{l+1}=$ $\cdots=z_{n}=0$ in $\mathbb{P}$, the degree of $G \mid \tilde{L}$ with respect to $s$ and $t$ is one, and we may 
write $G \mid \tilde{L}=h_{1} s+h_{2} t$ for some $h_{1}$ and $h_{2} \in \mathbb{k}\left[z_{0}, \ldots, z_{l}\right]$. Let $W$ be the subscheme of $L$ defined by $h_{1}=h_{2}=0$. Then $\operatorname{codim}(W, L)=2$ if $l \geq 1$, and $W=\emptyset$ if $l=0$, since $\tilde{X}$ is general and hence $h_{1}$ and $h_{2}$ are general. This together with $\phi^{-1}(L)=\tilde{L}$ implies that $\tilde{X} \rightarrow X$ is one-to-one and unramified at every point of $L \backslash W$ and also $X$ contains $L$. Thus $X \backslash W \subseteq \operatorname{Sm} X$.

To show (2), we will prove $W \subseteq \operatorname{Sing} X$. For a point $x \in W$, take general points $\tilde{x}_{1} \neq \tilde{x}_{2}$ of $\phi^{-1}(x) \cong \mathbb{P}^{1}$ so that $\tau \mid \tilde{X}: \tilde{X} \rightarrow \mathbb{P}^{1}$ is unramified at $\tilde{x}_{i}$. Set $\tilde{\mathbb{P}}_{i}^{n}=\tau^{-1}\left(\tau\left(\tilde{x}_{i}\right)\right)$ and $\mathbb{P}_{i}^{n}=\phi\left(\tilde{\mathbb{P}}_{i}^{n}\right)\left(\cong \tilde{\mathbb{P}}_{i}^{n}\right)$. To look at the dimension of the Zariski tangent space $\Theta_{x, X}$ to $X$ at $x$, consider $\Theta_{x, X}$ as a subspace of $\Theta_{x, \mathbb{P}^{N}}$. The space $\Theta_{x, X}$ contains the image of $\Theta_{\tilde{x}_{i}, \tilde{X}}(i=1,2)$ by the tangent map of $\phi$. Then $\operatorname{dim} \Theta_{\tilde{x}_{i}, \tilde{X}}=\operatorname{dim} \Theta_{\tilde{x}_{i}, \mathbb{P}}-1$ and $\Theta_{\tilde{x}_{i}, \tilde{\mathbb{P}}_{i}^{n}} \not \subset \Theta_{\tilde{x}_{i}, \tilde{X}}$. Since $\Theta_{\tilde{x}_{i}, \tilde{\mathbb{P}}_{i}^{n}} \cong \Theta_{x, \mathbb{P}_{i}^{n}}$ and $\Theta_{x, \mathbb{P}_{1}^{n}} \cap \Theta_{x, \mathbb{P}_{2}^{n}}=\Theta_{x, L}$ as subspaces of $\Theta_{x, \mathbb{P}^{N}}$, we have $\operatorname{dim} \Theta_{x, X} \geq 2(n-1)-l=$ $n+(n-l-2)$. If $n \geq l+3$, this means that $x \in \operatorname{Sing} X$. When $n=l+2$, we take another general point $\tilde{x}_{3} \in \phi^{-1}(x)$, and the same argument implies that $x \in \operatorname{Sing} X$.

Now (3) is easy. In fact, for $p \in \mathbb{P}^{1}, \tilde{X} \cap \tau^{-1}(p)$ is a hypersurface of degree $\mu \geq 2$ in $\tau^{-1}(p)\left(\cong \mathbb{P}^{n}\right)$, and hence $l(X \cap\langle x, y\rangle) \geq 3$ for every $x \in L \backslash W$ and general $y \in X$.

Finally we look at the relation between $\operatorname{Sing} X$ and the boundary of $\mathrm{C}(X)$.

Theorem 4.11. Let $X \subseteq \mathbb{P}^{N}$ be a nondegenerate, projective variety of dimension $n$ and codimension $e \geq 2$. Let $\Lambda$ be an irreducible component of the closure of $\mathrm{C}(X)$, of dimension $l$, which is necessarily linear by Theorem 5 .

(1) Assume $l \geq 2$. (Hence necessarily $n \geq l+1>l \geq 2$.) Then $\operatorname{dim} \operatorname{Sing} X \geq$ $n-2$, or $\operatorname{dim}(\Lambda \cap \operatorname{Sing} X) \geq l-2$.

(2) Assume $l \geq 3$. (Hence necessarily $n \geq l+1>l \geq 3$.) Then $\operatorname{dim}(\Lambda \cap$ Sing $X) \geq l-3$.

Proof. (1). Assume $\operatorname{dim}(\Lambda \cap \operatorname{Sing} X) \leq l-3$. We will show $\operatorname{dim} \operatorname{Sing} X \geq n-2$. By the assumption, there exists a 2-dimensional subspace $M$ of $\Lambda$ with $M \subseteq \operatorname{Sm} X$, and consequently $M \subseteq \mathrm{C}(X)$ by (4.2). Consider the linear projection $\pi_{M, X}: X \backslash M \rightarrow$ $\mathbb{P}^{N-3}$ from $M$ and let $\bar{X}$ be the closure of $\pi_{M, X}(X \backslash M)$. Let $x$ be a general point of $X$ and set $\bar{x}:=\pi_{M, X}(x)$. Let $X_{\bar{x}}$ be the closure of $\pi_{M, X}^{-1}(\bar{x})=\langle M, x\rangle \cap(X \backslash M)$ over $\bar{x}$. Since $M \subseteq \mathrm{C}(X)$, we have $\operatorname{dim} X_{\bar{x}}=2$ or 3 , and hence $\operatorname{dim} \bar{X}=n-2$ or $n-3$. In the latter, $X$ is the cone over $\bar{X}$ with vertex $\Lambda$ and hence $\Lambda \subseteq \operatorname{Sing} X$, a contradiction. Thus $\operatorname{dim} \bar{X}=n-2$. Hence $\operatorname{dim}\left\langle T_{\bar{x}}(\bar{X}), M\right\rangle=n+1$ and $T_{x^{\prime}}(X) \subseteq$ $\left\langle T_{\bar{x}}(\bar{X}), M\right\rangle$ for each $x^{\prime} \in X_{\bar{x}} \cap \operatorname{Sm} X$ (see (1.2.2)). By the theorem of tangencies ([23], (I.1.7)), $\operatorname{dim}\left(X_{\bar{x}} \cap \operatorname{Sing} X\right) \geq \operatorname{dim} X_{\bar{x}}-\operatorname{dim}\left\langle T_{\bar{x}}(\bar{X}), M\right\rangle+n-1=0$. This implies that $\langle M, x\rangle \cap(X \backslash M) \cap \operatorname{Sing} X \neq \emptyset$, since $M \subseteq \operatorname{Sm} X$ and $X_{\bar{x}} \backslash M=\langle M, x\rangle \cap(X \backslash M)$. By the generality of $x \in X$, Sing $X$ dominates $\bar{X}$, and consequently $\operatorname{dim} \operatorname{Sing} X \geq$ $n-2$, as required.

(2). Assume $\operatorname{dim}(\Lambda \cap \operatorname{Sing} X) \leq l-4$ to get a contradiction. There exists a 3-dimensional subspace $M^{\prime}$ of $\Lambda$ with $M^{\prime} \subseteq \operatorname{Sm} X$, and consequently $M^{\prime} \subseteq \mathrm{C}(X)$. Consider the linear projection $\pi_{M^{\prime}, X}: X \backslash M^{\prime} \rightarrow \mathbb{P}^{N-4}$ from $M^{\prime}$ and the closure $\bar{X}$ of $\pi_{M^{\prime}, X}\left(X \backslash M^{\prime}\right)$. For a general point $x \in X$, let $X_{\bar{x}}$ be the closure of $\pi_{M^{\prime}, X}^{-1}(\bar{x})$ over $\bar{x}:=\pi_{M^{\prime}, X}(x)$. By the same argument as in (1), $\operatorname{dim} X_{\bar{x}}=3, \operatorname{dim} \bar{X}=n-3$, and $\operatorname{dim}\left(X_{\bar{x}} \cap \operatorname{Sing} X\right) \geq 1$. Since $X_{\bar{x}} \subseteq\left\langle M^{\prime}, x\right\rangle$, we have $X_{\bar{x}} \cap \operatorname{Sing} X \cap M^{\prime} \neq \emptyset$, which contradicts $M^{\prime} \subseteq \operatorname{Sm} X$. 
Remark 4.12. In Example 4.10, by (4.11), we can relax the assumption $n \geq l+2$ to $n \geq l+1$ if $l \geq 2$. Indeed, $W \supseteq \operatorname{Sing} X$ without the assumption. Since $\operatorname{dim} W=l-2$, we have $\operatorname{dim}(\Lambda \cap \operatorname{Sing} X) \geq l-2$ by (4.11)(1). Since $W$ is irreducible by the generality of $\tilde{X}$ and hence by the generality of $h_{1}$ and $h_{2}$, we have $W=\operatorname{Sing} X=\Lambda \cap \operatorname{Sing} X$.

\section{REFERENCES}

1. A. Altman and S. Kleiman, Introduction to Grothendieck duality theory, Lecture Notes in Math., vol. 146, Springer, 1970. MR0274461 (43:224)

2. M.A. Bertin, On the regularity of varieties having an extremal secant line, J. Reine Angew. Math. 545 (2002), 167-181. MR1896101 (2003h:14078)

3. M.A. Bertin, On singular varieties having an extremal secant line, Comm. Algebra 34, no. 3 (2006), 893-909. MR.2208107 (2006k:14095)

4. A. Calabri and C. Ciliberto, On special projections of varieties: Epitome to a theorem of Beniamino Segre, Adv. Geom. 1 (2001), 97-106. MR1823955 (2002b:14064)

5. L. Chiantini, N. Chiarli, S. Greco, Bounding Castelnuovo-Mumford regularity for varieties with good general projections, J. Pure Appl. Algebra 152 no. 1-3 (2000), 57-64. MR1783985 (2001h:14058)

6. D. Eisenbud, S. Goto, Linear free resolutions and minimal multiplicity, J. Algebra 88 (1984), 89-133. MR741934 (85f:13023)

7. H. Flenner, L. O'Carroll, and W. Vogel, Joins and intersections, Springer Monog. Math., Springer-Verlag, 1999. MR.1724388 (2001b:14010)

8. T. Fujita, Classification theories of polarized varieties, London Math. Soc. Lecture Note Ser. 155, Cambridge Univ. Press, 1990. MR.1162108 (93e:14009)

9. L. Gruson, R. Lazarsfeld, C. Peskine, On a theorem of Castelnuovo, and the equations defining space curves, Invent. Math. 72 (1983), 491-506. MR704401 (85g:14033)

10. J. Harris, Algebraic Geometry, Graduate Texts in Math. 133, Springer-Verlag, 1992. MR 1182558 (93j:14001)

11. R. Hartshorne, Algebraic Geometry, Graduate Texts in Math. 52, Springer-Verlag., 1977. MR 0463157 (57:3116)

12. S. Kleiman, The enumerative theory of singularities, Nordic Summer School/NAVF Symposium in Mathematics, Oslo, August 5-25, 1976, pp. 297-396. MR0568897 (58:27960)

13. S. Kwak, Castelnuovo regularity for smooth subvarieties of dimensions 3 and 4, J. Algebraic Geom. 7 no. 1 (1998), 195-206.. MR1620706 (2000d:14043)

14. S. Kwak, Smooth projective varieties with extremal or next to extremal curvilinear secant subspaces, Trans. Amer. Math. Soc. 357 (2005), 3553-3566. MR.2146638 (2006e:14072)

15. R. Lazarsfeld, A sharp Castelnuovo bound for smooth surfaces, Duke Math. J. 55 (2) (1987), 423-429. MR894589 (89d:14007)

16. D. Mumford, Lectures on curves on an algebraic surface, Ann. of Math. Stud. 59, Princeton Univ. Press, 1966. MR0209285 (35:187)

17. D. Mumford, Varieties defined by quadratic equations, Questions on Algebraic Varieties (C.I.M.E., III Ciclo, Varenna, 1969) Edizioni Cremonese, Rome, 1970, pp. 29-100. MR0282975 (44:209)

18. A. Noma, Multisecant lines to projective varieties., Projective varieties with unexpected properties, Walter de Gruyter GmbH \& Co. KG, Berlin, 2005, pp. 349-359. MR2202263 (2006k:14099)

19. A. Noma, Multisecants to smooth projective varieties in arbitrary characteristic, Proc. Amer. Math. Soc. 137, no. 12 (2009), 3985-3990. MR2538558

20. B. Segre, On the locus of points from which an algebraic variety is projected multiply, Proceedings of the Phys.-Math. Soc. Japan Ser. III 18 (1936), 425-426.

21. B. Segre, Sul luogo dei punti da cui una data varietà algebrica iperspaziale è proiettata multiplamente, Atti primo Congr. Un. mat. Ital., 1937, pp. 264-269.

22. A. J. Sommese, J. Verschelde, C. W. Wampler, Numerical irreducible decomposition using projections from points on the components, Contemp. Math. 286 (2001), 37-51. MR1874270 (2002k:65089) 
23. F. L. Zak, Tangents and secants of algebraic varieties, Translations of Mathematical Monographs, vol. 127, 1993. MR.1234494 (94i:14053)

24. O. Zariski, Introduction to the problem of minimal models in the theory of algebraic surfaces, Publications of the Mathematical Society of Japan, no. 4, The Mathematical Society of Japan, Tokyo, 1958. MR0097403 (20:3872)

Department of Mathematics, Faculty of Education and Human Sciences, Yokohama NAtional University, YokOHAma 240-8501, JAPAN

E-mail address: noma@edhs.ynu.ac.jp 